\title{
Erratum
}

\section{Estimating optimal treatment regimes from a classification perspective}

\section{Baqun Zhang ${ }^{a *}$, Anastasios A. Tsiatis ${ }^{\mathrm{b}}$, Marie Davidian ${ }^{\mathrm{b}}$, Min Zhang ${ }^{\mathrm{c}}$ and}

\section{Eric Laber ${ }^{\mathrm{b}}$}

There was a sign typographical error in the equation on top of p. 107 of the original published version of Zhang et al. (2012).

The corrected equation is as follows:

$$
\begin{aligned}
\operatorname{AIPWE}(\eta) & =n^{-1} \sum_{i=1}^{n}\left\{\frac{\mathcal{C}_{\eta, i} Y_{i}}{\pi_{c}\left(X_{i} ; \eta, \hat{\gamma}\right)}-\frac{\mathcal{C}_{\eta, i}-\pi_{c}\left(X_{i} ; \eta, \hat{\gamma}\right)}{\pi_{c}\left(X_{i} ; \eta, \hat{\gamma}\right)} m\left(X_{i} ; \eta, \hat{\beta}\right)\right\} \\
& =n^{-1} \sum_{i=1}^{n}\left\{\frac{\mathcal{C}_{\eta, i}}{\pi_{c}\left(X_{i} ; \eta, \hat{\gamma}\right)} Y_{i}-\frac{\mathcal{C}_{\eta, i}-\pi_{c}\left(X_{i} ; \eta, \hat{\gamma}\right)}{\pi_{c}\left(X_{i} ; \eta, \hat{\gamma}\right)}\left[\mu\left(1, X_{i}, \hat{\beta}\right) g(X, \eta)+\mu\left(0, X_{i}, \hat{\beta}\right)\left\{1-g\left(X_{i}, \eta\right)\right\}\right]\right\} \\
& =n^{-1} \sum_{i=1}^{n}\left\{g\left(X_{i}, \eta\right) \hat{C}_{A I P W E}\left(X_{i}\right)\right\}+n^{-1} \sum_{i=1}^{n}\left\{\frac{1-A_{i}}{1-\pi\left(X_{i}, \hat{\gamma}\right)} Y_{i}+\frac{A_{i}-\pi\left(X_{i}, \hat{\gamma}\right)}{1-\pi\left(X_{i}, \hat{\gamma}\right)} \mu\left(0, X_{i}, \hat{\beta}\right)\right\} .
\end{aligned}
$$

\section{Reference}

Zhang, B, Tsiatis, AA, Davidian, M, Zhang, M \& Laber E (2012), 'Estimating optimal treatment regimes from a classification perspective', Stat, 1, 103-114. doi: 10.1002/sta4.11.

\footnotetext{
a Department of Preventive Medicine, Northwestern University, Chicago, IL 60611, USA

${ }^{b}$ Department of Statistics, North Carolina State University, Raleigh, NC, 27695-8203, USA

'Department of Biotatistics, University of Michigan, Ann Arbor, MI 48109-2029, USA

*Email: baqun.zhang@northwestern.edu
} 